\title{
Anterior pattern disease in adrenoleukodystrophy
}

\author{
Kanupriya Vijay • Tao Ouyang
}

Received: 17 December 2009/Revised: 11 March 2010/Accepted: 20 April 2010/Published online: 25 May 2010

(C) Springer-Verlag 2010

An 8-year-old boy presented in the emergency department with seizures. An MRI revealed symmetrical areas of T2 FLAIR hyperintensity and contrast enhancement involving the frontal white matter, the anterior commissure and the genu of corpus callosum (Figs. 1 and 2). There was no involvement of the parieto-occipital lobes or peritrigonal white matter, but there was a lesion in the splenium. There was sparing of the subcortical-U fibers. This was most consistent with the predominant frontal pattern of adrenoleukodystrophy (ALD), which was later confirmed with biochemical and cytogenetic examinations.

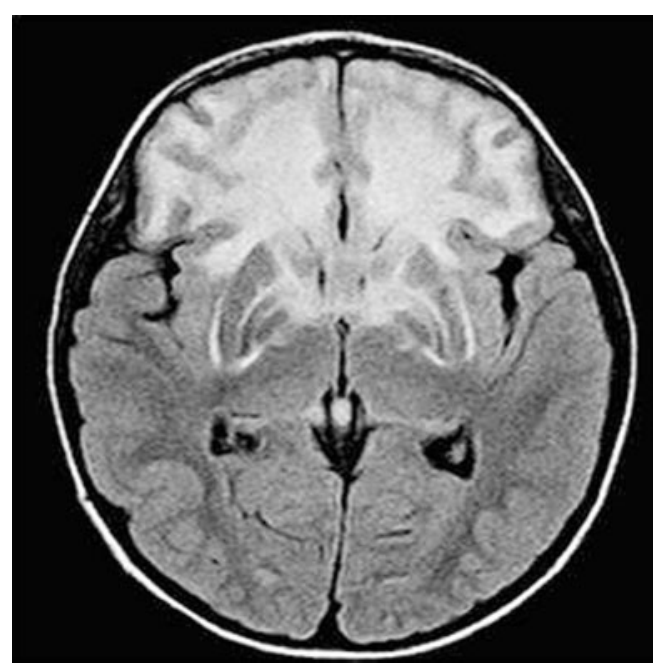

Fig. 1 T2 FLAIR axial image

K. Vijay $(\bowtie) \cdot$ T. Ouyang

Department of Radiology,

Milton S. Hershey Medical Center, Penn State University,

500 University Drive, Mail Code H066,

Hershey, PA 17033, USA

e-mail: kvijay@hmc.psu.edu

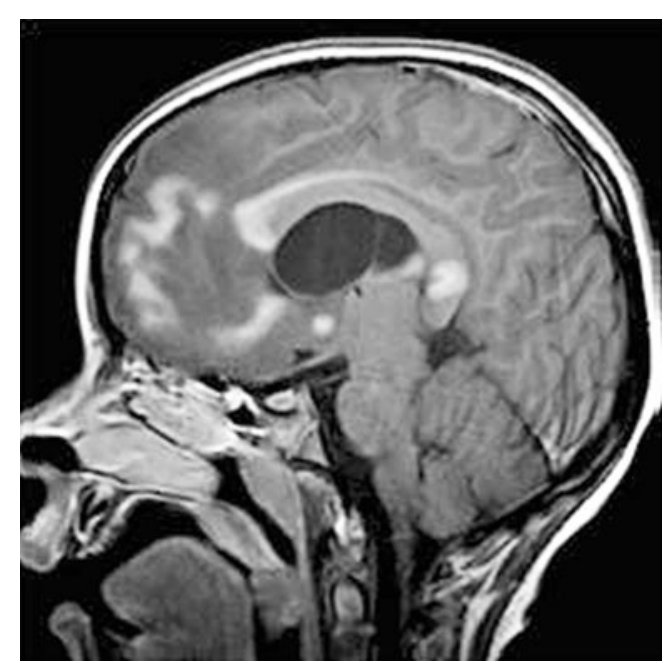

Fig. 2 Contrast-enhanced sagittal T1 image

Recently, five MR imaging patterns have been described in cerebral ALD [1]. The most common pattern $(66 \%)$ is characterized by confluent $\mathrm{T} 2$ prolongation of the deep parieto-occipital white matter and the visual pathway. The second most common pattern $(15 \%)$ is characterized by lesions in the deep anterior frontal white matter, genu and anterior body of the corpus callosum, also known as anterior pattern disease.

\section{Reference}

1. Kim JH, Kim HJ (2005) Childhood X-linked adrenoleukodystrophy: clinical-pathologic overview and MR imaging manifestations at initial evaluation and follow-up. Radiographics 25:619-631 